\title{
Construction of Multivariate Evaluation System of Mental Health Education Based on Big Data
}

\author{
Pengcheng Wei \\ Wuhan Polytechnic University, Wuhan, Hubei, 430023
}

Keywords: multivariate evaluation; mental health; big data; control charts; process capability

\begin{abstract}
With the development of big data concepts and technologies, big data has had an important impact on the reform and development of higher education. In order to improve the precision level of college students' mental health education, it is necessary to introduce the concept of big data into the field of mental health education in colleges and universities, analyze the data of mental health of college students in detail, and establish a mental health data feedback system model, which is helpful to put forward implementation path of data feedback.
\end{abstract}

\section{Introduction}

The psychological health education of college students refers to the way that college educators use psychological science to exert a positive influence on the psychological aspects of college students, promote their psychological adaptation, development, maturity, and maintain their mental health education practice activities. Mental health of college students is an important part of school moral education or ideological and political education. It is an important way to promote the healthy growth of college students, cultivate top-notch innovative talents, and is an important measure to fully implement the Party's education policy and build a strong human resource. Promote the Reform of Higher Education, Strengthen and Improve the Important Tasks of Ideological and Political Education for College Students [1]. In February 2011, the Ministry of Education issued a Notice on the Basic Construction Standards for the Psychological Health Education of College Students (Trial), emphasizing strengthening the "Construction of the College Students' Psychological Crisis Prevention and Intervention System”, including a survey of the mental health status of new students. With the attention of the state, various ordinary colleges and universities have successively carried out surveys on the mental health status of freshmen or all students. Research related to census has also become one of the research hotspots in the field of mental health. From the current general survey of university students' psychological health in our country, there are many researches on the status analysis of college students' psychological health census data and the strategies or suggestions for the problems found in the psychological survey of college students. However, the mental health of college students Research related to information sharing or feedback mechanisms is still relatively rare.

\section{Status of Mental Health Education in Data Use}

Mental health education in colleges and universities is also an important part of college moral education. Through the unremitting efforts of mental health workers, the mental health work system of the four major modules has been constructed, namely, mental health promotion, mental health curriculum teaching, psychological counseling, and psychological crisis intervention. However, looking back at the current state of data use in mental health education, many problems have arisen. The main performance is as follows.

First, the status of students' mental health data is fragmented and cannot be shared. Researchers are struggling to collect data on the mental health status of college students through relevant psychological scales. Due to limited data, the collected data can only be used for limited purposes. The results of analysis often cannot be shared with other people. As a result, the collected data is in an "island" state. In addition, mental health workers are unable to obtain relevant data from other 
departments and cannot fully grasp the psychological dynamics of students. In research and analysis, the actual value of a single data is actually very limited, and there is a one-sided danger. Only those data that cross the industry boundaries and the relevance of the data are highly accurate [2].

Second, there is still a considerable distance between data statistics and guidance practice in mental health education. Mental health education statistics are regarded as a statistical technique method that comprehensively analyzes causal relationships and variable relationships from a large number of disorganized student psychological health data or numerous complex educational phenomena, and infers the development and changes of students' mental health. At present, the statistical level of mental health education has developed considerably. Various statistical methods have emerged one after another. Structural equations and linear regression have become more mainstream in the analysis of mental health data. However, the significance of profoundly guiding practice is deteriorating. There are very limited opinions and suggestions on health work. The reason is that the abundant and wonderful college students have a simple quantitative relationship in which causality and variable relationships are evacuated into icy conditions. The actual meaning is greatly superficial, mechanized, and vulgarized. There is a phenomenon that focuses on "data analysis" and light "meaning interpretation." The resulting data value was ignored [3]. It should be said that this is not the intention of psychological health education statistics as a kind of scientific development. As an important part of mental health education work, it pursues the significance of guiding reality through the analysis of actual value through quantitative analysis because it is not only statistically analyzed. With the tool value attribute, it also has the ability to deeply interpret social complex relationships and guide social practice work. There is a huge gap between data mining and forecasting capabilities. Data mining analyzes the patterns that exist in the data and the relationships between the data. It emphasizes scientific analysis of a large amount of observed data, and finally accurately predicts the development of things. Different from physical measurement, psychological measurement is an indirect measurement of psychological traits. Coupled with limited sources of data and relatively simple data, the value of data is relatively limited, and the predictive effect is greatly reduced. Taking the psychological crisis intervention on campus as an example, psychological crisis intervention and recognition are usually unacceptable. One of the main reasons is that our current data forecasting ability is very weak. As a result, workers cannot grasp a large amount of information in time, interact with the intervention object in time, and make predictions and judgments in a timely manner. As a result, psychological crisis interventions often have failures [5]. In addition, at present, we do not pay enough attention to the forecasting ability of big data, and just as big data can provide practical help for the identification and intervention of students' psychological crisis.

\section{Path Selection of Big Data Technology in Mental Health Education}

At present, the psychological and behavioral data of college students mainly use the statistical analysis of clinical diagnostic scales, and more are collected by students' self-reports. This involves the students or the objective facts that intentionally or unintentionally do not report actual ideas. As a result, the collected data is distorted and it lacks certain scientificity. In the era of big data, students' psychological and behavioral data are not just scale data, but they should also include students' ubiquitous big data in schools, life, and the Internet. These include student's personal information and poor student management. Data, network information data, academic performance data, college students' psychological health assessment data, college students' health measurement data, school attendance data, and so on.

Under the support of big data technology and ideas, mental health education needs to break the "isolated island" situation of mental health data, realize horizontal and vertical information sharing, and improve the mental health work system. The mental health education service system breaks through the independent management system of "single fight" in which students share mental health information resources, which lays a solid foundation for the sharing of mental health resources, and enables related mental health educators to realize without limitation of time and space. The remote and cross-channel information retrieval and utilization of students' mental health information 
resources lays a solid foundation for better serving students. Of course, there are still some problems in the implementation. First, the management system needs to be optimized. This point, we can learn from New York University. The school set up data administrators and academic research offices. The data administrator is responsible for data collection from top to bottom, and the research office of the university is directly responsible for obtaining relevant data from the data. The two closely cooperate with each other, and both parties work together to ensure the accuracy and completeness of the school data [4]. To build a management system with mental health data with the characteristics of Chinese universities, we can implement a management system in which psychological counseling centers, student work centers, network centers, and faculty organizations work together and teachers and teachers work together. Mental health workers can directly extract data from databases. Data, data analysis and report writing. Second, establish a data protection mechanism. Mental health workers in colleges and universities need to use big data technology to deal with the students' mental health work. The premise is that the data is complete, accurate, large sample and so on. Guaranteeing the above characteristics of data often requires schools to ensure human, material and financial resources. To this end, school decision-making levels coordinate and balance data quality and cost. Finally, perfecting the data management system is also imperative. Establishing and perfecting the campus big data collection system requires clear responsibility for information collection, and gradually establishes related systems for collection, analysis, utilization, and use. Participating teachers and researchers are truly legal, confidential, and accurate in the use of big data. Records, collects and collects relevant information in a standardized and complete manner, and it is properly kept and updated in a timely manner. The interpretation of the results of big data must also be reasonable and reasonable. In short, the issue of data quality is not just a technical issue, but also needs to be guaranteed by the system.

Realization is a financial vocabulary, meaning cashless assets and securities are exchanged for cash. The realizing function of big data is to turn data into truly valuable behavior. The real value of big data is not to piece together the data, but to dig deeper and analyze it comprehensively to extract valuable psychology and behavior from the data [5]. Psychological crisis identification and intervention is a test standard for the realization of big data. Taking crisis intervention as an example, through analysis of big data, what are the main problems of specific types of students' psychological problems, academic performance, interpersonal relationships, learning motivation, class arrival rates, discipline violations, and psychological counseling? Dynamically grasp the psychological and behavioral changes of such groups, understand which parts of the population are high-risk groups, and which parts of the population need to focus on the crowd, and conduct crisis intervention in real time. Our mental health educators will be able to apply these data to the identification and intervention of students' psychological crisis. They will specifically provide counseling for this group of students before the corresponding psychological crisis, and eventually achieve the post-mortem adjustment and service of the psychological crisis. With targeted data analysis, our psychological crisis work can be targeted. The establishment of big data technology expands the network information channels to a certain extent and establishes an effective interaction mechanism so that college students can become active interventions from passive intervention. In addition, big data can realize the data realization function and can also provide practical programs for other curriculum education, mental health promotion, and psychological counseling.

\section{Conclusion}

With the development of big data concepts and technologies, Big Data has had an important impact on the reform and development of higher education. In order to improve the precision of college students' mental health education work, it is necessary to introduce the concept of big data into the field of mental health education in universities, and analyze the data of mental health of college students in detail, and establish a mental health data feedback system model, which is helpful to put forward Data feedback implementation path. 


\section{Acknowledgements}

This study was supported by "the general funding project for college Student Affairs in Hubei (item number 2016XGJPX3009)

\section{References}

[1] Qin Dianqi. Personal Knowledge Organization under the Integration and Big Data Concept[J]. Information Theory and Theory, 2014, (2).

[2] Li Yu. Big Data Concept and Library Big Data [J]. New Century Library, 2014, (6).

[3] Yu Changhong, Wang Yunwu. The goal, content and strategy of digital campus construction in the context of big data [J]. 2013, (10).

[4] Shan Yaojun. Research on the Information Management of College Students under the Background of Big Data[J]. Education and Occupation, 2014, (23).

[5] Wu Wei. Research on the Value Orientation of College Students Employment under the Background of Big Data [J]. Ideological and Theoretical Education, 2014, (2). 